\title{
On the Distance Identifying Set Meta-Problem and Applications to the Complexity of Identifying Problems on Graphs
}

\author{
Florian Barbero
}

LIRMM, Université de Montpellier, 161 rue Ada, 34095, Montpellier, France

florian.barbero@lirmm.fr

\section{Lucas Isenmann}

LIRMM, Université de Montpellier, 161 rue Ada, 34095, Montpellier, France

lucas.isenmann@lirmm.fr

\section{Jocelyn Thiebaut}

LIRMM, Université de Montpellier, 161 rue Ada, 34095, Montpellier, France

jocelyn.thiebaut@lirmm.fr

\begin{abstract}
Numerous problems consisting in identifying vertices in graphs using distances are useful in domains such as network verification and graph isomorphism. Unifying them into a meta-problem may be of main interest. We introduce here a promising solution named DisTANCE IDENTIFYING Set. The model contains Identifying Code (IC), Locating Dominating Set (LD) and their generalizations $r$-IC and $r$-LD where the closed neighborhood is considered up to distance $r$. It also contains Metric Dimension (MD) and its refinement $r$-MD in which the distance between two vertices is considered as infinite if the real distance exceeds $r$. Note that while $\mathrm{IC}=1-\mathrm{IC}$ and $\mathrm{LD}=1-\mathrm{LD}$, we have $\mathrm{MD}=\infty-\mathrm{MD}$; we say that $\mathrm{MD}$ is not local.

In this article, we prove computational lower bounds for several problems included in Distance Identifying Set by providing generic reductions from (Planar) Hitting Set to the meta-problem. We focus on two families of problem from the meta-problem: the first one, called bipartite gifted local, contains $r$-IC, $r$-LD and $r$-MD for each positive integer $r$ while the second one, called 1-layered, contains LD, MD and $r$-MD for each positive integer $r$. We have:

- the 1-layered problems are NP-hard even in bipartite apex graphs,

- the bipartite gifted local problems are NP-hard even in bipartite planar graphs,

- assuming ETH, all these problems cannot be solved in $2^{o(\sqrt{n})}$ when restricted to bipartite planar or apex graph, respectively, and they cannot be solved in $2^{o(n)}$ on bipartite graphs,

- even restricted to bipartite graphs, they do not admit parameterized algorithms in $2^{\mathcal{O}(k)} \cdot n^{\mathcal{O}(1)}$ except if $\mathrm{W}[0]=\mathrm{W}[2]$. Here $k$ is the solution size of a relevant identifying set.

In particular, METRIC Dimension cannot be solved in $2^{o(n)}$ under ETH, answering a question of Hartung in [20].
\end{abstract}

2012 ACM Subject Classification Mathematics of computing $\rightarrow$ Graph algorithms, Theory of computation $\rightarrow$ Problems, reductions and completeness

Keywords and phrases identifying code, resolving set, metric dimension, distance identifying set, parameterized complexity, W-hierarchy, meta-problem, hitting set

Digital Object Identifier 10.4230/LIPIcs.IPEC.2018.10

Related Version The full version of the article can be found in [4], https://arxiv.org/abs/ 1810.03868.

Acknowledgements We want to thank Stéphane Bessy and Anaël Grandjean for their precious advice concerning the correctness of the claims, and the structure of the article.

(c) (i) () Florian Barbero, Lucas Isenmann, and Jocelyn Thiebaut;

13th International Symposium on Parameterized and Exact Computation (IPEC 2018).

Editors: Christophe Paul and Michał Pilipczuk; Article No. 10; pp. 10:1-10:14

Leibniz International Proceedings in Informatics 


\section{Introduction and Corresponding Works}

Problems consisting in identifying each element of a combinatorial structure with a hopefully small number of elements have been widely investigated. Here, we study a meta identification problem which generalizes three of the most well-known identification problems in graphs, namely Identifying Code (IC), Locating Dominating Set (LD) and Metric Dimension (MD). These problems are used in network verification [3, 5], fault-detection in networks [22, 28], graph isomorphism [2] or logical definability of graphs [23]. The versions of these problems in hypergraphs have been studied under different names in [6], [7] and [8].

Given a graph $G$ with vertex set $V$, the classical identifying sets are defined as follows:

- IC: Introduced by Karposky et al. [22], a set $C$ of vertices of $G$ is said to be an identifying code if none of the sets $N[v] \cap C$ are empty, for $v \in V$ and they are all distinct.

- LD: Introduced by Slater [25, 26], a set $C$ of vertices of $G$ is said to be a locatingdominating set if none of the sets $N[v] \cap C$ are empty, for $v \in V \backslash C$ and they are all distinct. When not considering the dominating property $(N[v] \cap C$ may be empty), these sets have been studied in [2] as distinguing sets and in [23] as sieves.

- MD: Introduced independently by Harary et al. [18] and Slater [24], a set $C$ of vertices of $G$ is said to be a resolving set if $C$ contains one vertex from each connected component of $G$ and, for every distinct vertices $u$ and $v$ of $G$, there exists a vertex $w$ of $C$ such that $d(w, u) \neq d(w, v)$. The metric dimension of $G$ is the minimum size of its resolving sets.

The corresponding minimization problems of the previous identifying sets are defined as follows: given a graph $G$, compute a suitable set $C$ of minimal size, if one exists. In this paper, we mainly focus on the computational complexity of these minimization problems.

Known results. A wide collection of NP-hardness results has been proven for the problems.

For IC and LD, the minimization problems are indeed NP-hard [10, 11]. Charon et al. showed the NP-hardness when restricted to bipartite graphs [9], while Auger showed it for planar graphs with arbitrarily large girth [1]. For trees, there exists a linear algorithm [25].

The MD problem is also NP-hard, even when restricted to Gabriel unit disk graphs [17, 21]. Epstein et al. [14] showed that MD is polynomial on several classes as trees, cycles, cographs, partial wheels, and graphs of bounded cyclomatic number, but it remains NP-hard on split graphs, bipartite graphs, co-bipartite and line graphs of bipartite graphs. Additionally, Diaz et al. [12] proved a quite tight separation: the problem is polynomial on outerplanar graphs whereas it remains NP-hard on bounded degree planar graphs.

In a recent publication, Foucaud et al. [16] also proved the NP-hardness of the three problems restricted to interval graphs and permutation graphs.

These notions may be considered under the parameterized point of view; see [13] for a comprehensive study of Fixed Parameter Tractability (FPT). In the following, the parameter $k$ is chosen as the solution size of a distance identifying set.

For IC and LD, the parameterized problems are clearly FPT since the number of vertices of a positive instance is bounded by $2^{k}+k$ ( $k$ vertices may characterize $2^{k}$ neighbors).

Such complexity is not likely to be achievable in the case of MD, since it would imply $\mathrm{W}[2]=\mathrm{FPT}(=\mathrm{W}[0])$. Indeed, Hartung et al. [19, 20] showed MD is W[2]-hard for bipartite subcubic graphs. The problem is however FPT on families of graphs with degree $\Delta$ growing with the number of vertices because the size $k$ of a resolving set must satisfy $\log _{3}(\Delta)<k$. Finally, Foucaud et al. [16] provided a FPT algorithm on interval graphs. 


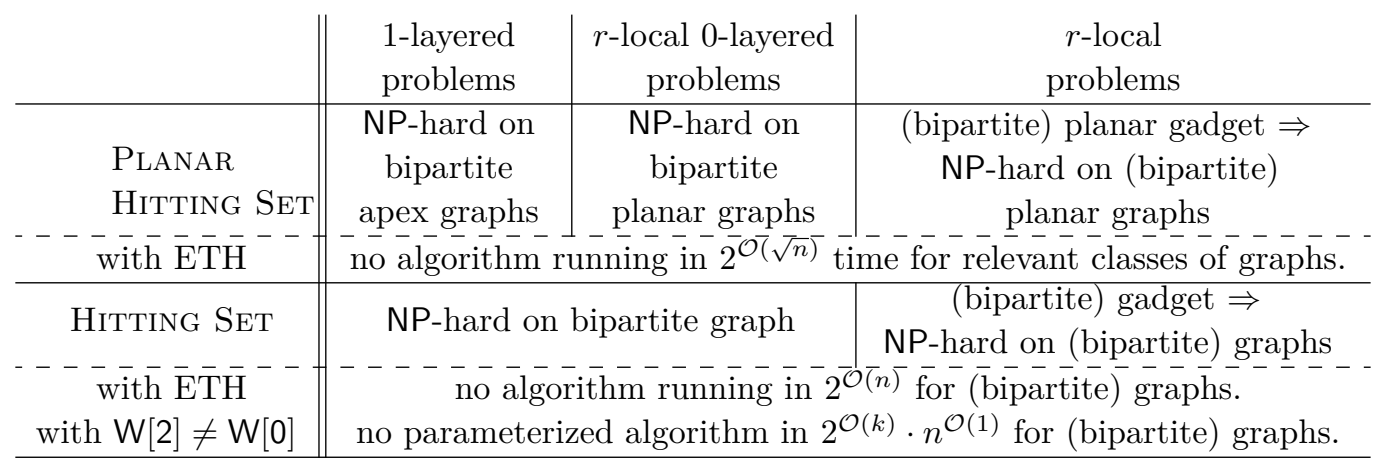

Figure 1 The computational lower bounds implied by our generic reductions.

Our contributions. In order to unify the previous minimization problems, we introduce the concept of distance identifying functions. Given a distance identifying function $f$ and a value $r$ as a positive integer or infinity, the Distance IDENTIFy ING SET meta-problem consists in finding a minimal sized $r$-dominating set which distinguishes every couple of vertices of an input graph thanks to the function $f$. Here, we mainly focus on two natural subfamilies of problems of Distance IDEnTIFying SET named local, in which a vertex cannot discern the vertices outside of its $i$-neighborhood, for $i$ a fixed positive integer, and 1-layered, where a vertex is able to separate its open neighborhood from the distant vertices.

With this approach, we obtain several computational lower bounds for problems included in Distance Identifying Set by providing generic reductions from (Planar) Hitting SET to the meta-problem. The reductions rely on the set/element-gadget technique, the noteworthy adaptation of the clause/variable-gadget technique from SAT to HitTing SET.

As we provide a 1-layered generic gadget, the 1-layered reductions operate without condition. For local problems, the existence of a local gadget is not always guaranteed. Thus, a local reduction operates only if a local gadget is provided. However, the local planar reduction is slightly more efficient than its 1-layered counterpart: it indeed implies computational lower bounds for planar graphs whereas the 1-layered reduction requires an auxiliary apex, limiting the consequences to apex graphs.

The reductions in general graphs are designed to exploit the W[2]-hardness of HitTinG SET parameterized by the solution size $k_{H S}$ of an hitting set, hereby using:

- Theorem 1 (folklore). Let $n_{H S}$ and $m_{H S}$ be the number of elements and sets of an HiTTING SET instance, and $k_{H S}$ be its solution size. A parameterized problem with parameter $k$ admitting a reduction from HitTING SET verifying $k=\mathcal{O}\left(k_{H S}+\log \left(n_{H S}+m_{H S}\right)\right)$ does not have a parameterized algorithm running in $2^{\mathcal{O}(k)} \cdot n^{\mathcal{O}(1)}$ time except if $\mathrm{W}[2]=\mathrm{FPT}$.

Proof. Given a reduction from Hitting SeT to a parameterized problem $\Pi$ such that the reduced parameter satisfies $k=\mathcal{O}\left(k_{H S}+\log \left(n_{H S}+m_{H S}\right)\right)$ and the size of the reduced instance verifies $n=\left(n_{H S}+m_{H S}\right)^{\mathcal{O}(1)}$, an algorithm for $\Pi$ of running time $2^{\mathcal{O}(k)} \cdot n^{\mathcal{O}(1)}$ is actually an algorithm for HitTing SET of running time $2^{\mathcal{O}\left(k_{H S}\right)} \cdot\left(n_{H S}+m_{H S}\right)^{\mathcal{O}(1)}$, meaning that Hitting SET is FPT, a contradiction to its W[2]-hardness (otherwise W[2] = FPT).

Hence, as each gadget contributes to the resulting solution size of a distance identifying set, we set up a binary compression of the gadgets to limit their number to the logarithm order. From the best of our knowledge, this merging gadgets technique has never been employed.

The organization of the paper is as follows. After a short reminder of the computational properties of Hitting SET, Section 2 contains the definitions of distance identifying functions and sets, allowing us to precise the computation lower bounds we obtain. Section 3 designs 
the supports of the reductions as distance identifying graphs and compressed graph. Finally, the gadgets needed for the reductions to apply are given in Section 4.

\section{Definition of the Meta-Problem and Related Concepts}

\subsection{Preliminaries}

Notations. Throughout the paper, we consider simple non oriented graphs.

Given a positive integer $n$, the set of positive integers smaller than $n$ is denoted by $\llbracket n \rrbracket$. By extension, we define $\llbracket \infty \rrbracket=\mathbb{N}_{>0} \cup\{\infty\}$. Given two vertices $u, v$ of a graph $G$, the distance between $u$ and $v$ corresponds to the number of vertices in the shortest path between $\mathrm{u}$ and $\mathrm{v}$ and is denoted $d(u, v)$. The open neighborhood of $u$ is denoted by $N(u)$, its closed neighborhood is $N[u]=N(u) \cup\{u\}$, and for a value $r \in \llbracket \infty \rrbracket$, the $r$-neighborhood of $u$ is $N_{r}[u]$, that is the set of vertices at distance less than $r+1$ of $u$. For $r=\infty$, the $\infty$-neighborhood of $u$ is the set of vertices in the same connected component than $u$. We recall that a subset $D$ of $V$ is called a $r$-dominating set of $G$ if for all vertices $u$ of $V$, the set $N_{r}[u] \cap D$ is non-empty. Thus an $\infty$-dominating set of $G$ contains at least a vertex for each connected component of $G$.

Given two subsets $X$ and $Y$ of $V$, the distance $d(X, Y)$ corresponds to the value $d(X, Y)=$ $\min \{d(x, y) \mid x \in X, y \in Y\}$. For a vertex $u$, we will also use $d(u, X)$ and $d(X, u)$, defined similarly. The symmetric difference between $X$ and $Y$ is denoted by $X \Delta Y$, and the 2-combination of a set $X$ is denoted $\mathcal{P}_{2}(X)$

Given two graphs $G=\left(V_{G}, E_{G}\right)$ and $H=\left(V_{H}, E_{H}\right), H$ is an induced subgraph of $G$ if $V_{H} \subseteq V_{G}$ and for all vertices $u$ and $v$ of $V_{H},(u, v) \in E_{G}$ if and only if $(u, v) \in E_{H}$. We denote $H=G\left[V_{H}\right]$ and $V_{G} \backslash V_{H}$ by $V_{G \backslash H}$. Symmetrically, $G$ is an induced supergraph of $H$.

\section{The (Planar) Hitting Set problem}

Consider a universe of $n$ elements denoted $\Omega=\left\{u_{i} \mid i \in \llbracket n \rrbracket\right\}$ and a set of $m$ non-empty subsets of $\Omega$ denoted $\mathcal{S}=\left\{S_{i} \mid i \in \llbracket m \rrbracket\right\}$ such that every element belongs to at least a subset. Then, a subset of $\Omega$ intersecting every set of $\mathcal{S}$ is called an hitting set of $\mathcal{S}$ :

HitTing SeT

Input: A universe $\Omega$ and a set $\mathcal{S}$ of non-empty subsets of $\Omega$ whose union covers $\Omega$.

Output: A minimal-sized hitting set $C$ of $\mathcal{S}$, i.e. a subset of $\Omega$ satisfying $\forall S_{i} \in \mathcal{S}, S_{i} \cap C \neq \emptyset$. The parameterized version Hitting $\operatorname{Set}(\mathrm{k})$ decides if there exists a hitting set of size $k$.

- Theorem 2 (R.G. Downey and M.R Fellows [13]). Hitting SeT cannot be solved in $2^{o(n)}$ time under ETH even if $m=\mathcal{O}(n)$. Moreover, Hitting $\operatorname{Set}(k)$ is W[2]-hard.

Hitting SeT may be translated into a dominating problem on bipartite graphs. Given an instance $(\Omega, \mathcal{S})$ of Hitting Set, let us define $\phi(\Omega, \mathcal{S})=\left(V_{\Omega} \cup V_{\mathcal{S}}, E\right)$ as the bipartite graph of size $n+m$ such that for each $i \in \llbracket n \rrbracket$, there exists a vertex $v_{i}^{\Omega}$ in $V_{\Omega}$, for each $j \in \llbracket m \rrbracket$, there exists a vertex $v_{j}^{\mathcal{S}}$ in $V_{\mathcal{S}}$, and the edge $\left(v_{i}^{\Omega}, v_{j}^{\mathcal{S}}\right)$ is present in $E$ if and only if the element $u_{i}$ belongs to the subset $S_{j}$. Henceforth, a hitting set of $\mathcal{S}$ is equivalent to a subset $C$ of $V_{\Omega}$ that dominates $V_{\mathcal{S}}$. We call $\phi(\Omega, \mathcal{S})$ the associated graph of $(\Omega, \mathcal{S})$ :

Planar Hitting Set

Input: An instance $(\Omega, \mathcal{S})$ of HitTing SET such that $\phi(\Omega, \mathcal{S})$ is planar.

Output: A hitting set $C$ of $\mathcal{S}$ of minimal size.

We also consider the parameterized version Planar Hitting Set(k) of the latter problem. 
- Theorem 3 (folklore). There exists a reduction from SAT to PlanAR Hitting Set $(n)$ producing associated graphs of quadratic size in the number $n$ of variables of the instances of SAT. Thus Planar Hitting Set cannot be solved in $2^{o(\sqrt{n})}$ under ETH even if $m=\mathcal{O}(n)$.

Sketch of proof. A linear reduction from SAT to Hitting SET is known. To guarantee the planarity of $\phi(\Omega, \mathcal{S})$, we apply the reduction on a restriction of SAT named SEPARATE Simple PlanaR SAT which is not solvable in $2^{o(\sqrt{n})}$ under ETH. See [27].

\subsection{The Distance Identifying Set meta-problem}

Given a graph $G=(V, E)$ and $r \in \llbracket \infty \rrbracket$, the classical identifying sets may be rewritten:

- $r$-IC: a subset $C$ of $V$ is a $r$-identifying code of $G$ if it is an $r$-dominating set and for every distinct vertices $u, v$ of $V$, a vertex $w$ in $C$ verifies $w \in N_{r}[u] \Delta N_{r}[v]$.

- $r$-LD: a subset $C$ of $V$ is a $r$-locating dominating set of $G$ if it is an $r$-dominating set and for every distinct vertices $u, v$ of $V$, a vertex $w$ in $C$ verifies $w \in\left(N_{r}[u] \Delta N_{r}[v]\right) \cup\{u, v\}$.

- $r$-MD: a subset $C$ of $V$ is a $r$-resolving set of $G$ if it is an $r$-dominating set and for every distinct vertices $u, v$ of $V$, a vertex $w$ in $C$ verifies $w \in N_{r}[u] \cup N_{r}[v]$ and $d(u, w) \neq d(v, w)$.

A pattern clearly appears: the previous identifying sets only deviate on the criterion that the vertex $w$ must verify. The pivotal idea is to consider an abstract version of the criterion which does not depend on the input graph. Hence:

Definition 4 (identifying function). A function $f$ of type: $G \rightarrow\left(V \times \mathcal{P}_{2}(V) \rightarrow\{\right.$ true, false $\left.\}\right)$, is called an identifying function. Given three vertices $u, v$ and $w$ of a graph $G$ such that $u \neq v$, we write $f_{G}[w](u, v)$ to get the resulting boolean. The notation $\mathcal{P}_{2}(V)$ implies that $f_{G}$ is symmetric, that is $f_{G}[w](u, v)=f_{G}[w](v, u)$.

We need to require some useful properties on identifying functions to produce generic results. By mimicking the classical identifying sets, the main property we consider is that a vertex cannot distinguish two vertices at the same distance from it. Then:

- Definition 5 (distance function). A distance identifying function $f$ is an identifying function such that for every graph $G$ and all vertices $u, v$ and $w$ of $G$ with $u \neq v$ :

( $\alpha) f_{G}[w](u, v)$ is false when $d(u, w)=d(v, w)$.

Besides this mandatory criterion, we suggest two paradigms related to the neighborhood of a vertex. Let $i \in \llbracket \infty \rrbracket$. First, we may restrain the range of a vertex to its $i$-neighborhood: a vertex should not distinguish two vertices if they do not lie in its $i$-neighborhood but it should always distinguish them whenever exactly one of them lies to that $i$-neighborhood. Reciprocally, we may ensure that a vertex could distinguish the vertices of its $i$-neighborhood: a vertex should distinguish a vertex belonging to its $i$-neighborhood from all the other vertices, assuming the distances are different. Formally, we have:

- Definition 6 ( $i$-local function). For $i \in \llbracket \infty \rrbracket$, an $i$-local identifying function $f$ is an identifying function such that for every graph $G$ and all vertices $u, v, w$ of $G$ with $u \neq v$ :

$\left(\beta_{1}\right) f_{G}[w](u, v)$ is true when $d(u, w) \leq i<d(v, w)$ or, symmetrically, $d(v, w) \leq i<d(u, w)$. $\left(\beta_{2}\right) f_{G}[w](u, v)$ is false when $i<\min \{d(u, w), d(v, w)\}$.

- Definition 7 ( $i$-layered function). For $i \in \llbracket \infty \rrbracket$, an $i$-layered identifying function $f$ is an identifying function such that for every graph $G$ and all vertices $u, v, w$ of $G$ with $u \neq v$ :

$(\gamma) f_{G}[w](u, v)$ is true when $\min \{d(u, w), d(v, w)\} \leq i$ and $d(u, w) \neq d(v, w)$. 
In the following, given an identifying function $f$ and three vertices $u, v, w$ of a graph $G$, we say that $w f$-distinguishes $u$ and $v$ if and only if $f_{G}[w](u, v)$ is true. By extension, given three vertex sets $C, X$ and $Y$ of $G$, we say that $C f$-distinguishes $X$ and $Y$ if for every $u$ in $X$ and $v$ in $Y$, either $u=v$ or there exists $w$ in $C$ verifying $f_{G}[w](u, v)$. Finally, a graph $G$ of vertex set $V$ is $f$-distinguished by $C$ when $C f$-distinguishes $V$ and $V$.

We are now ready to define the Distance Identifying Set meta-problem.

- Definition $8((f, r)$-distance identifying set). For a distance identifying function $f$ and $r \in \llbracket \infty \rrbracket$, a $(f, r)$-distance identifying set of a graph $G$ is an $r$-dominating set of $G$ that $f$-distinguishes $G$.

Distance Identifying SeT

Input: A distance identifying function $f$ and $r \in \llbracket \infty \rrbracket$. A graph $G$.

Output: A $(f, r)$-distance identifying set of $G$ of minimal size, if one exists.

Given a distance identifying function $f$ and $r \in \llbracket \infty \rrbracket$ as inputs of the meta-problem, the resulting problem is called $(f, r)$-Distance IDEnTIFYING SET and denoted $(f, r)$-DIS. The problem $(f, r)$-DIS is said to be $i$-layered when the function $f$ is $i$-layered, and it is said to be $i$-local when $f$ is $i$-local and $r=i$. A problem is local if it is $i$-local for an integer $i$. Recall that our local reductions need a local gadget to operate: the subfamilies of local problems admitting a (bipartite) local gadget is called (bipartite) gifted local. We do not need to define gifted 1-layered as every 1-layered problem admits a 1-layered gadget. We also consider the parameterized version Distance Identifying $\operatorname{Set}(k)$.

\subsection{Detailed Computational Lower Bounds}

Using the Distance Identifying Set meta-problem, we get the following lower bounds:

- Theorem 9. For each 1-layered distance identifying function $f$ and every $r \in \llbracket \infty \rrbracket$, the $(f, r)$-Distance Identifying SET problem restricted to bipartite apex graphs is NP-hard, and does not admit an algorithm running in $2^{\mathcal{O}(\sqrt{n})}$ time under ETH.

- Theorem 10. The (bipartite) gifted local problems restricted to (bipartite) planar graphs are NP-hard, and do not admit an algorithm running in $2^{\mathcal{O}(\sqrt{n})}$ time under ETH.

- Theorem 11. For each r-local 0-layered distance identifying function $f,(f, r)$-DIS restricted to bipartite planar graphs is NP-hard, and cannot be solved in $2^{\mathcal{O}(\sqrt{n})}$ under ETH.

- Theorem 12. Let $f, g$ and $h$ be distance identifying functions such that $f$ is 1-layered, $g$ is q-local 0-layered and $h$ is $p$-local and admits a local (bipartite) gadget. Let $r \in \llbracket \infty \rrbracket$. The $(f, r)-,(g, q)-$ and $(h, p)$ - DIS problems are NP-hard, and do not admit:

- algorithms running in $2^{o(n)}$ time, except if ETH fails,

- parameterized algorithms running in $2^{\mathcal{O}(k)} \cdot n^{\mathcal{O}(1)}$ time, except if $W[2]=$ FPT.

The parameter $k$ denotes here the solution size of a relevant distance identifying set.

All bounds still hold in the bipartite case (whenever the gadget associated with $h$ is bipartite).

As a side result, the 1-layered general reduction answers a question of Hartung in [20]:

- Corollary 13. Under ETH, Metric Dimension cannot be solved in $2^{o(n)}$.

Finally, notice that the parameterized lower bound from Theorem 12 may be complemented by an elementary upper bound inspired from the kernel of IC and LD of size $2^{k}+k$ :

- Proposition 14. For every $r$-local distance identifying function $f$, the $(f, r)$-Distance IDENTIFYING SET problem has a kernel of size $(r+1)^{k}+k$ where $k$ is the solution size. Therefore, it admits a naive parameterized algorithm running in $\mathcal{O}\left(n^{k+3}\right) \in \mathcal{O}^{*}\left(r^{\left(k^{2}\right)}\right)$ time. 


\section{The Supports of the Reductions for Distance Identifying Set}

\subsection{The Distance Identifying Graphs}

Consider the associated graph $\phi(\Omega, \mathcal{S})$ as defined in Section 2.1. The differences between the Distance Identifying Set meta-problem and the dominating problem related to associated graphs actually raise two issues for a reduction based on these latter notions to be effective on Distance Identifying Set. First, contrarily to the dominating problem where a vertex may only discern its close neighborhood, the meta-problem may allow a vertex to discern further than its direct neighborhood. In that case, we cannot certify that a vertex $v_{i}^{\Omega}$ does not distinguish a vertex $v_{j}^{\mathcal{S}}$ when $u_{i}$ is not in $S_{j}$, the adjacency not remaining a sufficient argument. Secondly, one may object that a vertex $v_{i}^{\Omega}$ formally has to distinguish a $\operatorname{vertex} v_{j}^{\mathcal{S}}$ from another vertex, but that distinguishing a single vertex is not defined.

To circumvent these problems, we suggest the following fix: rather than producing a single vertex for each $S_{j} \in \mathcal{S}$, the set $V_{\mathcal{S}}$ may contain two vertices $v_{j}^{\mathcal{S}}$ and $\bar{v}_{j}^{\mathcal{S}}$. Then, the role of $v_{i}^{\Omega}$ would be to distinguish them if and only if $u_{i} \in S_{j}$. To ensure that the vertex $v_{i}^{\Omega}$ distinguishes $v_{j}^{\mathcal{S}}$ and $\bar{v}_{j}^{\mathcal{S}}$ when $u_{i} \in S_{j}$, we may use the properties $\left(\beta_{1}\right)$ and $(\gamma)$ of Definition 6 and 7 for the $r$-local and 1-layered problems, respectively. Precisely, when $u_{i} \in S_{j}, v_{i}^{\Omega}$ should be at distance $r$ to $v_{j}^{\mathcal{S}}$ (with $r=1$ in the 1-layered cases) while $\bar{v}_{j}^{\mathcal{S}}$ should not be in the $r$-neighborhood of $v_{i}^{\Omega}$. Similarly, to ensure that $v_{i}^{\Omega}$ cannot distinguish $v_{j}^{\mathcal{S}}$ and $\bar{v}_{j}^{\mathcal{S}}$ when $u_{i} \notin S_{j}$, we may use properties $(\alpha)$ or $\left(\beta_{2}\right)$ of Definitions 5 and 6 . Hence, when $u_{i} \notin S_{j}, v_{j}^{\mathcal{S}}$ should not be in the $r$-neighborhood of $v_{i}^{\Omega}$, or $d\left(v_{i}^{\Omega}, v_{j}^{\mathcal{S}}\right)$ and $d\left(v_{i}^{\Omega}, \bar{v}_{j}^{\mathcal{S}}\right)$ should be equal.

That fix fairly indicates how to initiate the transformation of the associated graphs in order to deliver an equivalence between a hitting set formed by elements of $\Omega$ and the vertices of a distance identifying set included in $V_{\Omega}$. However, it is clearly not sufficient since we also have to distinguish the couples of vertices of $V_{\Omega}$ for which nothing is required. To solve that problem, we suggest to append to each vertex of the associated graph a copy of some gadget with the intuitive requirement that the gadget is able to distinguish the close neighborhood of its vertices from the whole graph. We introduce the notion of $B$-extension:

- Definition 15 (B-extension). Let $H=\left(V_{H}, E_{H}\right)$ be a connected graph, and $B \subseteq V_{H}$. An induced supergraph $G=\left(V_{G}, E_{G}\right)$ is said to be a $B$-extension of $H$ if it is connected and for every vertex $v$ of $V_{G \backslash H}$, the set $N(v) \cap V_{H}$ is either equal to $\emptyset$ or $B$.

A vertex $v$ of $V_{G \backslash H}$ such that $N(v) \cap V_{H}=B$ is said to be $B$-adjacent. The $B$-extensions of $H$ such that $V_{G \backslash H}$ contains exactly a $B$-adjacent vertex or two $B$-adjacent (but not neighbors) vertices are called the $B$-single-extension and the B-twin-extension of $H$, respectively.

Here, the border $B$ makes explicit the connections between a copy of a gadget $H$ and a vertex outside the copy. In particular, a $B$-single-extension is formed by a gadget with its related vertex $v_{i}^{\Omega}$, while a $B$-twin-extension contains a gadget with its two related vertices $v_{j}^{\mathcal{S}}$ and $\bar{v}_{j}^{\mathcal{S}}$. Piecing all together, we may adapt the associated graphs to the meta-problem:

- Definition 16 (distance identifying graph). Let $\left(\Omega=\left\{u_{i} \mid i \in \llbracket n \rrbracket\right\}, \mathcal{S}=\left\{S_{i} \mid i \in \llbracket m \rrbracket\right\}\right)$ be an instance of HitTing Set. Let $H$ be a connected graph, $B$ a subset of its vertices, and $r$ a positive integer. The $(H, B, r)$-distance identifying graph $\Phi[H, B, r](\Omega, \mathcal{S})$ is as follows.

- for each $i \in \llbracket n \rrbracket$, the graph $\Phi[H, B, r](\Omega, \mathcal{S})$ contains as induced subgraph a copy $H_{i}^{\Omega}$ of $H$ together with a $B_{i}^{\Omega}$-adjacent vertex $v_{i}^{\Omega}$, where $B_{i}^{\Omega}$ denotes the copy of $B$.

- similarly, for each $j \in \llbracket m \rrbracket$, the graph $\Phi[H, B, r](\Omega, \mathcal{S})$ contains a copy $H_{j}^{\mathcal{S}}$ of $H$ together with two $B_{j}^{\mathcal{S}}$-adjacent vertices $v_{j}^{\mathcal{S}}$ and $\bar{v}_{j}^{\mathcal{S}}$; the latter vertices are not adjacent.

- finally, for each $S_{j} \in \mathcal{S}$ and each $u_{i} \in S_{j}, v_{i}^{\Omega}$ is connected to $v_{j}^{\mathcal{S}}$ by a path of $r-1$ vertices denoted $l_{i, j}^{k}$ with $d\left(v_{i}^{\Omega}, l_{i, j}^{k}\right)=k$ for each $k \in \llbracket r-1 \rrbracket$. 

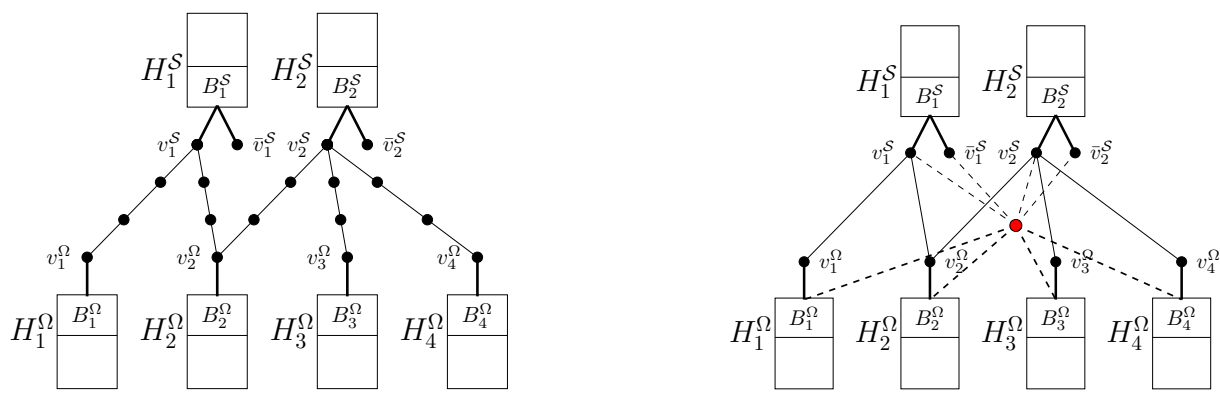

Figure $2 \mathrm{~A}(H, B, 3)$-distance identifying graph and a $(H, B)$-apex distance identifying graph built on the planar instance formed by $\Omega=\{1,2,3,4\}$ and $\mathcal{S}=\{\{1,2\},\{2,3,4\}\}$.

When the problem is not local, we prefer the following identifying graph:

- Definition 17 ( $(H, B)$-apex distance identifying graph). An $(H, B)$-apex distance identifying graph $\Phi^{*}[H, B](\Omega, \mathcal{S})$ is the union of a $(H, B, 1)$-distance identifying graph with an additional vertex $a$ called apex such that:

- for each $u_{i} \in \Omega$, the apex $a$ is $B_{i}^{\Omega}$-adjacent to $H_{i}^{\Omega}$.

- for each $S_{j} \in \mathcal{S}$, the apex $a$ is adjacent to $v_{j}^{\mathcal{S}}$ and $\bar{v}_{j}^{\mathcal{S}}$.

- Proposition 18. Given an instance $(\Omega, \mathcal{S})$ of Planar Hitting Set where $|\Omega|=n$, $|\mathcal{S}|=m$, the graphs $G=\Phi[H, B, r](\Omega, \mathcal{S})$ and $G^{\prime}=\Phi^{*}[H, B](\Omega, \mathcal{S})$

- are connected and have size bounded by $(|H|+2 r)(n+m)$, (with $r=1$ for $\left.G^{\prime}\right)$,

- may be built in polynomial time in their size,

- are bipartite if the $B$-single extension of $H$ is bipartite,

- are respectively planar and an apex graph if the B-twin-extension of $H$ is planar.

Having defined the (apex) distance identifying graphs, the main effort to obtain generic reduction from Planar Hitting Set is done. We now define relevant gadgets:

Definition $19((f, r)$-gadgets $)$. Let $f$ be a distance identifying function and $r \in \llbracket \infty \rrbracket$. Let $H=\left(V_{H}, E_{H}\right)$ be a connected graph, and $B, C$ be two subsets of $V_{H}$. We said that the triple $(H, B, C)$ is a $(f, r)$-gadget if for every $B$-extension $G$ of $H$ :

$\left(p_{h}\right) C f$-distinguishes $V_{H}$ and $V_{G}$.

$\left(p_{b}\right) C f$-distinguishes $N_{B}$ and $V_{G \backslash H} \backslash N_{B}$, where $N_{B}$ is the set of $B$-adjacent vertices of $G$.

$\left(p_{d}\right) C$ is an $r$-dominating set of $G\left[V_{H} \cup N_{B}\right]$.

$\left(p_{s}\right)$ For all $(f, r)$-distance identifying set $S$ of $G,|C| \leq\left|S \cap V_{H}\right|$.

- Definition 20 (local gadgets). A $(f, r)$-gadget is a local gadget, if $f$ is a $r$-local identifying function with $r \neq \infty$, and $\left(p_{l}\right)$ : for every $k \in \llbracket r \rrbracket$, there exists $c \in C$ such that $d(c, B)=k-1$.

Consistently, we say that a $(f, r)$-gadget $(H, B, C)$ is bipartite if the $B$-single-extension of $H$ is bipartite, and that it is planar if the $B$-twin-extension of $H$ is planar.

- Theorem 21. Let $(\Omega, \mathcal{S})$ be an instance of HitTing SET such that $|\Omega|=n>1,|\mathcal{S}|=m$. Let $(H, B, C)$ be a $(f, r)$-gadget for a 1-layered identifying function $f$ and let $\left(H^{\prime}, B^{\prime}, C^{\prime}\right)$ be a local $(g, q)$-gadget. The following propositions are equivalent:

- there exists a hitting set of $\mathcal{S}$ of size $k$.

- there exists a $(f, r)$-distance identifying set of $\Phi^{*}[H, B](\Omega, \mathcal{S})$ of size $k+|C|(n+m)$.

- there exists a $(g, q)$-distance identifying set of $\Phi\left[H^{\prime}, B^{\prime}, q\right](\Omega, \mathcal{S})$ of size $k+\left|C^{\prime}\right|(n+m)$. 
Proof of Theorem 21. We focus on the equivalence between the first and second items.

Suppose first that $P$ is a hitting set of $(\Omega, \mathcal{S})$ of size $k$. By denoting $C_{i}^{\Omega}$ and $C_{j}^{\mathcal{S}}$ the copies of $C$ associated to the copies $H_{i}^{\Omega}$ and $H_{j}^{\mathcal{S}}$ of $H$, we suggest the following set $I$ of size $k+|C|(n+m)$ as a $(f, r)$-distance identifying set of $G=\Phi^{*}[H, B](\Omega, \mathcal{S})$ :

$$
I=\left\{v_{i}^{\Omega}: u_{i} \in P\right\} \cup \bigcup_{i \in \llbracket n \rrbracket} C_{i}^{\Omega} \cup \bigcup_{j \in \llbracket m \rrbracket} C_{j}^{\mathcal{S}} .
$$

Recall that by construction, $G$ is a $B_{i}^{\Omega}$-extension of $H_{i}^{\Omega}$ (respectively $B_{j}^{\mathcal{S}}$-extension of $H_{j}^{\mathcal{S}}$ ) for any $i \in \llbracket n \rrbracket$ (respectively $j \in \llbracket m \rrbracket$ ). This directly implies that $I$ is an $r$-dominating set of $G$. Indeed, the condition $\left(p_{d}\right)$ of Definition 19 implies that $C_{i}^{\Omega}$ (respectively $C_{j}^{\mathcal{S}}$ ) $r$-dominates $H_{i}^{\Omega}$ plus $v_{i}^{\Omega}$ (respectively of $H_{j}^{\mathcal{S}}$ plus $v_{j}^{\mathcal{S}}, \bar{v}_{j}^{\mathcal{S}}$ ). The remaining apex is also $r$-dominated by any $C_{i}^{\Omega}$, as it is $B_{i}^{\Omega}$-adjacent for every $i \in \llbracket n \rrbracket$.

We now have to show that $I f$-distinguishes $G$. We begin with the vertices of the gadget copies because the condition $\left(p_{h}\right)$ implies that $C_{i}^{\Omega} \subseteq I f$-distinguishes the vertices of $H_{i}^{\Omega}$ and $G$ for every $i \in \llbracket n \rrbracket$, and $I f$-distinguishes the vertices of $H_{j}^{\mathcal{S}}$ and $G$ for every $j \in \llbracket m \rrbracket$. Thereby, we only have to study the vertices of the form $v_{i}^{\Omega}, v_{j}^{\mathcal{S}}, \bar{v}_{j}^{\mathcal{S}}$, and the apex $a$ (there is no vertex of the form $l_{i, j}^{k}$ in an apex distance identifying graph). To distinguish them, we use the condition $\left(p_{b}\right)$. Recall that $n>1$. Then, for each distinct $i, i^{\prime} \in \llbracket n \rrbracket$, we have:

- $v_{i}^{\Omega}$ is $B_{i}^{\Omega}$-adjacent but not $B_{i^{\prime}}^{\Omega}$-adjacent,

- $a$ is both $B_{i}^{\Omega}$-adjacent and $B_{i^{\prime}}^{\Omega}$-adjacent,

- a vertex of the form $v_{j}^{\mathcal{S}}$ or $\bar{v}_{j}^{\mathcal{S}}$ is neither $B_{i}^{\Omega}$-adjacent nor $B_{i^{\prime}}^{\Omega}$-adjacent.

Enumerating the relevant $i$ and $i^{\prime}$, we deduce that every couple of vertices is distinguished except when they are both of the form $v_{j}^{\mathcal{S}}$ or $\bar{v}_{j^{\prime}}^{\mathcal{S}}$ for $j, j^{\prime} \in \llbracket m \rrbracket$. But we may distinguish $v_{j}^{\mathcal{S}}$ or $\bar{v}_{j^{\prime}}^{\mathcal{S}}$ for distinct $j, j^{\prime}$ by applying $\left(p_{b}\right)$ on $H_{j}^{\mathcal{S}}$.

It remains to distinguish $v_{j}^{\mathcal{S}}$ and $\bar{v}_{j}^{\mathcal{S}}$ for $j \in \llbracket m \rrbracket$. We now use the fact that $P$ is a hitting set for $(\Omega, \mathcal{S})$. By definition of a hitting set, for any set $S_{j} \in \mathcal{S}$, there exists a vertex $u_{i} \in P$ such that $u_{i} \in S_{j}$. We observe that $d\left(v_{i}^{\Omega}, v_{j}^{\mathcal{S}}\right)=1<d\left(v_{i}^{\Omega}, \bar{v}_{j}^{\mathcal{S}}\right)$ by construction of $G$ and that $v_{i}^{\Omega} \in I$ by definition of $I$. Since $f$ is 1-layered, $I f$-distinguishes $v_{j}^{\mathcal{S}}$ and $\bar{v}_{j}^{\mathcal{S}}$.

In the other direction, assume that $I$ is a distance identifying set of $G$ of size $k+|C|(n+m)$. As every set of $S$ is not empty, we may define a function $\varphi: \llbracket m \rrbracket \rightarrow \llbracket n \rrbracket$ such that $u_{\varphi(j)} \in S_{j}$.

We suggest the following set $P$ as an hitting set of $\mathcal{S}$ of size at most $k$ :

$$
P=\left\{u_{i} \in \Omega \mid v_{i}^{\Omega} \in I\right\} \cup\left\{u_{\varphi(j)} \in \Omega \mid v_{j}^{\mathcal{S}} \in I \text { or } \bar{v}_{j}^{\mathcal{S}} \in I\right\}
$$

We claim that the only vertices that may $f$-distinguish $v_{j}^{\mathcal{S}}$ and $\bar{v}_{j}^{\mathcal{S}}$ are themselves and the vertices $v_{i}^{\Omega}$ such that $u_{i} \in S_{j}$. To prove so, we apply propriety $(\alpha)$ of Definition 5 :

- the apex $a$ verifies $d\left(a, v_{j}^{\mathcal{S}}\right)=1=d\left(a, \bar{v}_{j}^{\mathcal{S}}\right)$

- a vertex $v_{i}^{\Omega}$ such that $u_{i} \notin S_{j}$ verifies $d\left(v_{i}^{\Omega}, v_{j}^{\mathcal{S}}\right)=3=d\left(v_{i}^{\Omega}, \bar{v}_{j}^{\mathcal{S}}\right)$

- a vertex $v$ of $H_{i}^{\Omega}$ verifies $d\left(v, v_{j}^{\mathcal{S}}\right)=2+d\left(v, B_{i}^{\Omega}\right)=d\left(v, \bar{v}_{j}^{\mathcal{S}}\right)$

- a vertex $v$ of $H_{j^{\prime}}^{\mathcal{S}}$ with $j \neq j^{\prime}$ verifies $d\left(v, v_{j}^{\mathcal{S}}\right)=3+d\left(v, B_{j}^{\mathcal{S}}\right)=d\left(v, \bar{v}_{j}^{\mathcal{S}}\right)$

- both $v_{j}^{\mathcal{S}}$ and $\bar{v}_{j}^{\mathcal{S}}$ are $B_{j}^{\mathcal{S}}$-adjacent, so they are at the same distance of any vertex of $H_{j}^{\mathcal{S}}$.

We deduce that $v_{j}^{\mathcal{S}}$ and $\bar{v}_{j}^{\mathcal{S}}$ are $f$-distinguished only if either one on them belongs to $I$ (in that case $u_{\varphi(j)} \in P \cap S_{j}$ ) or there exists $v_{i}^{\Omega} \in I$ such that $u_{i} \in S_{j}$ (and then $u_{i} \in P \cap S_{j}$ ).

It remains to show that $|P| \leq k$. By the condition $\left(p_{s}\right)$ of Definition 19, we know that $\left|I \cap V_{H_{i}^{\Omega}}\right| \geq\left|C_{i}^{\Omega}\right|$ and $\left|I \cap V_{H_{j}^{\mathcal{S}}}\right| \geq\left|C_{j}^{\mathcal{S}}\right|$ for any $i \in \llbracket n \rrbracket$ and $j \in \llbracket m \rrbracket$, implying

$$
k=|I|-|C|(n+m) \geq \sum_{i \in[n]}\left|I \cap\left\{v_{i}^{\Omega}\right\}\right|+\sum_{j \in[m]}\left|I \cap\left\{v_{j}^{\mathcal{S}}, \bar{v}_{j}^{\mathcal{S}}\right\}\right| \geq \sum_{v_{i}^{\Omega} \in I} 1+\sum_{I \cap\left\{v_{j}^{\mathcal{S}}, \bar{v}_{j}^{\mathcal{S}}\right\} \neq \emptyset} 1=|P|
$$

The equivalence between the first and third points is proven in [4]. 


\subsection{Binary Compression of Gadgets}

The Theorem 21 is a powerful tool to get reductions, in particular in the planar cases. However, the number of involved gadgets does not allow to use Theorem 1. This limitation is due to the uses of a gadget per vertex to identify in the distance identifying graphs. Using power set, we may obtain a better order: given $k$ gadgets, we may identify $2^{k}-1$ vertices (we avoid to identify a vertex with the empty subset of gadgets). Thus, we will consider binary representations of integers as sequences of bits, with weakest bit at last position. For a positive integer $n$, we define the integer $\lg _{n}=1+\left\lfloor\log _{2}(n)\right\rfloor$ and introduce a new graph:

Definition $22\left((H, B, r)\right.$-compressed graph). Let $\left(\Omega=\left\{u_{i} \mid i \in \llbracket n \rrbracket\right\}, \mathcal{S}=\left\{S_{i} \mid i \in \llbracket m \rrbracket\right\}\right)$ be an instance of Hitting SET. Let $H$ be a connected graph, $B$ be a subset of its vertices, and $r$ be a positive integer. The $(H, B, r)$-compressed graph $\Psi[H, B, r](\Omega, \mathcal{S})$ is defined as follows. $\Psi[H, B, r](\Omega, \mathcal{S})$ contains as induced subgraphs $\lg _{n+1}$ copies of $H$ denoted $H_{i}^{\Omega}$ for $i \in \llbracket \lg _{n+1} \rrbracket$ and $\lg _{m}$ another copies of $H$ denoted $H_{j}^{\mathcal{S}}$ for $j \in \llbracket \lg _{m} \rrbracket$. Then:

- for each $j \in \llbracket m \rrbracket$, we add two non-adjacent vertices $v_{j}^{\mathcal{S}}$ and $\bar{v}_{j}^{\mathcal{S}}$. They are $B_{k}^{\mathcal{S}}$-adjacent for each $k \in \llbracket \lg _{m} \rrbracket$ such that the $k^{t h}$ bit of the binary representation of $j$ is 1 .

- for each $i \in \llbracket n \rrbracket$, we add $r$ vertices denoted $l_{i}^{j-1}$ with $j \in \llbracket r \rrbracket$ to form a fresh path such that $d\left(v_{i}^{\Omega}, l_{i}^{j-1}\right)=j-1$ where $v_{i}^{\Omega}=l_{i}^{0}$. We make $v_{i}^{\Omega} B_{k}^{\Omega}$-adjacent for each $k \in \llbracket \lg _{n+1} \rrbracket$ such that the $k^{t h}$ bit of the binary representation of $i$ is 1 .

- for each $S_{j} \in \mathcal{S}$ and each $u_{i} \in S_{j}$, we add the edge $\left(l_{i}^{r-1}, v_{j}^{\mathcal{S}}\right)$.

- we add $r$ vertices denoted $a^{j-1}$ with $j \in \llbracket r \rrbracket$ to form a path such that $d\left(a^{0}, a^{j-1}\right)=j-1$. The vertex $a^{0}$ is $B_{k}^{\Omega}$-adjacent for every $k \in \llbracket \lg _{n+1} \rrbracket$, and we add the edges $\left(a^{r-1}, v_{j}^{\mathcal{S}}\right)$ and $\left(a^{r-1}, \bar{v}_{j}^{\mathcal{S}}\right)$ for each $j \in \llbracket m \rrbracket$.

By definition of $\lg _{n+1}$, for every $i \in \llbracket n \rrbracket$, one of the last $\lg _{n+1}$ bits of the binary representation of $i$ is 0 . So, $a^{0}$ has a distinct characterization in the power set formed by the gadgets $H_{i}^{\Omega}$.

- Proposition 23. The graph $\Psi[H, B, r](\Omega, \mathcal{S})$ built on an instance $(\Omega, \mathcal{S})$ of HitTING SET

- is connected and has size at most $|H|\left(\lg _{n+1}+\lg _{m}\right)+r(n+1)+2 m$, where $|\Omega|=n$, $|\mathcal{S}|=m$

- may be built in polynomial time in its size,

- is bipartite if the $B$-single extension of $H$ is bipartite.

- Theorem 24. Let $(\Omega, \mathcal{S})$ be an instance of HitTing SET such that $|\Omega|=n,|\mathcal{S}|=m$. Let $(H, B, C)$ be a $(f, r)$-gadget for a 1-layered identifying function $f$ and let $\left(H^{\prime}, B^{\prime}, C^{\prime}\right)$ be a local $(g, q)$-gadget. The following propositions are equivalent:

- there exists a hitting set of $\mathcal{S}$ of size $k$.

- there exists a $(f, r)$-distance identifying set of $\Psi[H, B, 1](\Omega, \mathcal{S})$ of size $k+|C|\left(\lg _{n+1}+\lg _{m}\right)$.

- there exists a $(q, r)$-distance identifying set of $\Psi\left[H^{\prime}, B^{\prime}, q\right](\Omega, \mathcal{S})$ of size $k+\left|C^{\prime}\right|\left(\lg _{n+1}+\lg _{m}\right)$.

\section{On Providing Gadgets to Establish Generic Reductions}

In this section, we finalize the reductions by furnishing some gadgets and combining them with the suitable theorems and propositions from Section 3. We directly define the gadgets.

- Definition 25 (The 1-layered gadget). Let $H$ be the bipartite planar graph such that:

- Its ten vertices are denoted $b, \bar{b}, u_{1}, \bar{u}_{1}, u_{2}, \bar{u}_{2}, v_{1}, \bar{v}_{1}, v_{2}$ and $\bar{v}_{2}$,

- The vertices $u_{1}, u_{2}, \bar{u}_{1}$ and $\bar{u}_{2}$ form a cycle as well as the vertices $v_{1}, v_{2}, \bar{v}_{1}$ and $\bar{v}_{2}$.

- The vertices $b$ and $\bar{b}$ are adjacent to $u_{1}, \bar{u}_{1}, v_{1}$ and $\bar{v}_{1}$. 


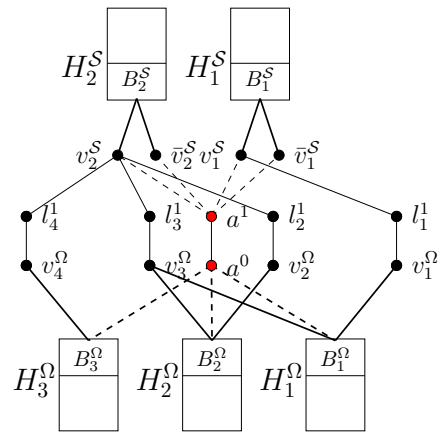

Figure $3 \mathrm{~A}(\mathrm{H}, \mathrm{B}, 2)$-compressed graph built with $\Omega=\{1,2,3,4\}$ and $\mathcal{S}=\{\{1,2\},\{2,3,4\}\}$.

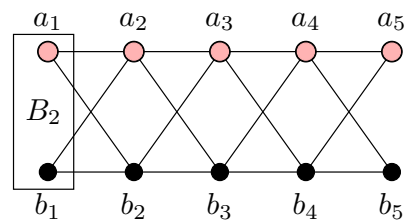

Figure 5 The 2-local 0-layered gadget. $C_{2}$ contains the colored vertices.

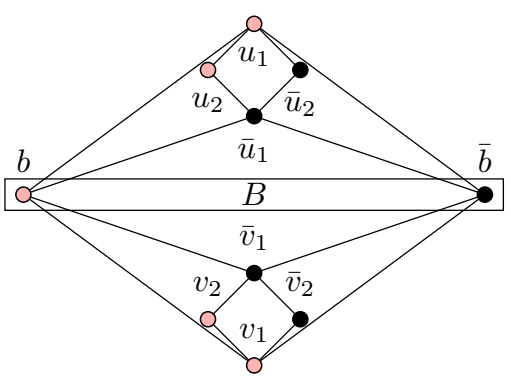

Figure 4 The 1-layered gadget $(H, B, C)$. $C$ contains the colored vertices.

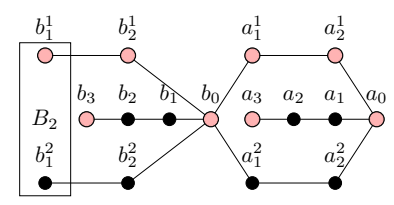

Figure 6 The 2-IC gadget.

$C_{2}$ contains the colored vertices.

We define the sets $B=\{b, \bar{b}\}$ and $C=\left\{b, u_{1}, u_{2}, v_{1}, v_{2}\right\}$.

The triple $(H, B, C)$ is called the 1-layered gadget (see Fig. 4).

- Definition 26 (The $r$-local 0-layered gadget). Given an integer $r>1$ (respectively $r=1$ ), let $H_{r}$ be the bipartite planar graph of size $4 r+2$ (respectively 8 ) such that:

- its vertices are denoted $a_{i}$ and $b_{i}$ for $i \in \llbracket 2 r+1 \rrbracket$ (respectively $\left.i \in \llbracket 4 \rrbracket\right)$,

- for each $i \in \llbracket 2 r \rrbracket$ (respectively $i \in \llbracket 3 \rrbracket$ ), both $a_{i}$ and $b_{i}$ are adjacent to $a_{i+1}$ and $b_{i+1}$ We define the sets $B_{r}=\left\{a_{1}, b_{1}\right\}$ and $C_{r}=\left\{a_{i} \mid i \in \llbracket 2 r+1 \rrbracket\right\}$ (respectively $C_{1}=$ $\left.\left\{a_{1}, a_{2}, a_{3}, a_{4}\right\}\right)$. The triple $\left(H_{r}, B_{r}, C_{r}\right)$ is called the $r$-local 0-layered gadget (see Fig. 5).

For each positive integer $r, r$-LD and $r$-MD are $r$-local 0 -layered problems, whereas $r$-IC is not 0 -layered. We define a specific gadget for this remaining problem.

Definition 27 (The $r$-IC gadget). Given a positive integer $r$, let $H_{r}$ be the bipartite planar graph of size $6 r+4$ such that:

- its vertices are denoted $a_{i-1}$ and $b_{i-1}$ for $i \in \llbracket r+2 \rrbracket$, and $a_{i}^{j}$ and $b_{i}^{j}$ for $i \in \llbracket r \rrbracket$ and $j \in \llbracket 2 \rrbracket$.

We also denote $a_{0}$ as $a_{r+1}^{1}$ and $a_{r+1}^{2}$ and we denote $b_{0}$ as $b_{r+1}^{1}, b_{r+1}^{2}, a_{0}^{1}$ and $a_{0}^{2}$.

- the edges are all included in the six following paths

- from $a_{0}$ to $a_{r+1}$ such that $d\left(a_{0}, a_{i}\right)=i$ for $i \in \llbracket r+1 \rrbracket$.

- from $b_{0}$ to $b_{r+1}$ such that $d\left(b_{0}, b_{i}\right)=i$ for $i \in \llbracket r+1 \rrbracket$.

- from $a_{0}^{1}$ to $a_{r+1}^{1}$ such that $d\left(a_{0}^{1}, a_{i}^{1}\right)=i$ for $i \in \llbracket r+1 \rrbracket$.

- from $a_{0}^{2}$ to $a_{r+1}^{2}$ such that $d\left(a_{0}^{2}, a_{i}^{2}\right)=i$ for $i \in \llbracket r+1 \rrbracket$.

- from $b_{1}^{1}$ to $b_{r+1}^{1}$ such that $d\left(b_{1}^{1}, b_{i}^{1}\right)=i-1$ for $i \in \llbracket r+1 \rrbracket$.

- from $b_{1}^{2}$ to $b_{r+1}^{2}$ such that $d\left(b_{1}^{2}, b_{i}^{2}\right)=i-1$ for $i \in \llbracket r+1 \rrbracket$.

We define the sets $B_{r}=\left\{b_{1}^{1}, b_{1}^{2}\right\}$ and $C_{r}=\left\{a_{r+1}, b_{r+1}\right\} \cup \underset{i \in \llbracket r+1 \rrbracket}{\bigcup}\left\{a_{i}^{1}, b_{i}^{1}\right\}$.

The triple $\left(H_{r}, B_{r}, C_{r}\right)$ is called the $r$-IC gadget (see Fig. 6).

As expected, we have the following propositions: 
- Proposition 28. The 1-layered gadget is a bipartite planar $(f, r)$-gadget for any 1-layered distance identifying function $f$ and $r \in \llbracket \infty \rrbracket$.

- Proposition 29. Given a positive integer $r$, the $r$-local 0-layered gadget is a local bipartite planar $(f, r)$-gadget for every $r$-local 0 -layered distance identifying function $f$.

- Proposition 30. Given a positive integer $r$, the $r$-IC gadget is a local bipartite planar $(f, r)$-gadget for the identifying function $f$ associated with $r$-IC, where $f_{G}[w](u, v)=$ true if $w \in N_{r}[u] \Delta N_{r}[v]$ for relevant inputs $G, u, v$ and $w$.

With Propositions 28 to 30 , we can now prove the Theorems 9 to 12 . We focus here on 1-layered distance identifying functions and show the other reductions in [4].

Proof of Theorems $\mathbf{9}$ and $\mathbf{1 2}$ for each 1-layered identifying function $f$ and $r \in \llbracket \infty \rrbracket$.

We first suggest a reduction from Planar Hitting Set to $(f, r)$-DIS based on the bipartite planar 1-layered gadget $(H, B, C)$. Let $(\Omega, \mathcal{S})$ be an instance of Planar Hitting Set with $|\Omega|=n$ and $|\mathcal{S}|=m$ such that $m=\mathcal{O}(n)$. According to Proposition 18, the bipartite apex graph $G=\Phi^{*}[H, B](\Omega, \mathcal{S})$ has size $n^{\prime}$ linear in $n+m=\mathcal{O}(n)$ and may be built in polynomial-time in its size. Recall that $(H, B, C)$ is a $(f, r)$-gadget by Proposition 28. By Theorem 21, $G$ admits a $(f, r)$-distance identifying set of size $k^{\prime}=k+|C|(n+m)$ if an only if $\mathcal{S}$ admits an hitting set of size $k$. Thus, an algorithm solving $(f, r)$-DIS in $2^{o\left(\sqrt{n^{\prime}}\right)}$ would solve Planar Hitting Set in time $2^{o(\sqrt{n})}$, a contradiction to Theorem 3 (assuming ETH).

We adapt the previous argumentation to get a reduction from HitTing SET to $(f, r)$-DIS, the instance $(\Omega, \mathcal{S})$ belonging now to the Hitting Set problem. According to Proposition 23 , the bipartite graph $G=\Psi[H, B, 1](\Omega, \mathcal{S})$ has size $n^{\prime}$ linear in $n+m=\mathcal{O}(n)$ and may also be built in polynomial-time in its size. By Theorem $24, G$ admits a $(f, r)$-distance identifying set of size $k^{\prime}=k+|C|\left(\lg _{n+1}+\lg _{m}\right)$ if an only if $\mathcal{S}$ admits an hitting set of size $k$. Thus, an algorithm solving $(f, r)$-DIS in $2^{o\left(n^{\prime}\right)}$ would solve HitTing SET in time $2^{o(n)}$, contradicting Theorem 2 when assuming ETH. Moreover, a parameterized algorithm solving $(f, r)$-DIS in $2^{\mathcal{O}(k)} \cdot{n^{\prime \mathcal{O}(1)}}$ would be in contradiction with Theorem 1 when assuming W[2] $\neq$ FPT.

\section{Conclusion}

In this paper, we showed generic tools to analysis identifying problems and their computational lower bounds. This study opens some new questions. First of all, we observe that our toolbox does not contain a $r$-local gadget. Does one exist? Furthermore, there is still a gap between the computational lower bound provided by Theorem 12 and the elementary upper bound from Proposition 14 in the local cases. We wonder if local problems may be solved in $k^{\mathcal{O}(k)} \cdot n^{\mathcal{O}(1)}$. Notice that a polynomial kernel would imply such a complexity (but the reciprocal is not true). For non-local problems, an FPT upper bound is globally unknown. In particular, $W[2]$-hard problems like MD cannot admit FPT algorithms unless $W[2]=$ FPT. Then, which non-local problem is W[2]-hard? We mention that we actually get a FPT reduction from HitTing SET to some scarce non-local problems (however including MD) proving their $\mathrm{W}[2]$-hardness, but the family of involved problems is not precise nor wide. Nevertheless, we remark that most of our reductions may be generalized to the oriented version of Distance IDENTIFYING SET sometimes even for the strongly connected graphs -this is due to the fact that the paths in our distance identifying graphs and gadgets may often be seen as oriented-. Thus, we inform the community that the oriented version of MD (studied for Cayley graphs in [15]) remains W[2]-hard. 


\section{References}

1 David Auger. Minimal identifying codes in trees and planar graphs with large girth. Eur. J. Comb., 31(5):1372-1384, 2010. doi:10.1016/j.ejc.2009.11.012.

2 László Babai. On the Complexity of Canonical Labeling of Strongly Regular Graphs. SIAM J. Comput., 9(1):212-216, 1980. doi:10.1137/0209018.

3 Evangelos Bampas, Davide Bilò, Guido Drovandi, Luciano Gualà, Ralf Klasing, and Guido Proietti. Network verification via routing table queries. J. Comput. Syst. Sci., 81(1):234248, 2015. doi:10.1016/j.jcss.2014.06.003.

4 Florian Barbero, Lucas Isenmann, and Jocelyn Thiebaut. On the Distance Identifying Set meta-problem and applications to the complexity of identifying problems on graphs. ArXiv e-prints, October 2018. arXiv:1810.03868.

5 Zuzana Beerliova, Felix Eberhard, Thomas Erlebach, Alexander Hall, Michael Hoffmann, Matús Mihalák, and L. Shankar Ram. Network Discovery and Verification. IEEE Journal on Selected Areas in Communications, 24(12):2168-2181, 2006. doi:10.1109/JSAC. 2006. 884015.

6 Béla Bollobás and Alex D. Scott. On separating systems. Eur. J. Comb., 28(4):1068-1071, 2007. doi:10.1016/j.ejc.2006.04.003.

7 J.A Bondy. Induced subsets. Journal of Combinatorial Theory, Series B, 12(2):201-202, 1972. doi:10.1016/0095-8956(72)90025-1.

8 Emmanuel Charbit, Irène Charon, Gérard D. Cohen, Olivier Hudry, and Antoine Lobstein. Discriminating codes in bipartite graphs: bounds, extremal cardinalities, complexity. Adv. in Math. of Comm., 2(4):403-420, 2008. doi:10.3934/amc.2008.2.403.

9 Irène Charon, Olivier Hudry, and Antoine Lobstein. Minimizing the size of an identifying or locating-dominating code in a graph is NP-hard. Theor. Comput. Sci., 290(3):2109-2120, 2003. doi:10.1016/S0304-3975(02)00536-4.

10 Gérard D. Cohen, Iiro S. Honkala, Antoine Lobstein, and Gilles Zémor. On identifying codes. In Codes and Association Schemes, Proceedings of a DIMACS Workshop, Piscataway, New Jersey, USA, November 9-12, 1999, pages 97-110, 1999.

11 Charles J Colbourn, Peter J Slater, and Lorna K Stewart. Locating dominating sets in series parallel networks. Congr. Numer, 56:135-162, 1987.

12 Josep Díaz, Olli Pottonen, Maria J. Serna, and Erik Jan van Leeuwen. On the Complexity of Metric Dimension. In Algorithms - ESA 2012 - 20th Annual European Symposium, Ljubljana, Slovenia, September 10-12, 2012. Proceedings, pages 419-430, 2012. doi:10. 1007/978-3-642-33090-2_37.

13 Rodney G. Downey and Michael R. Fellows. Parameterized Complexity. Monographs in Computer Science. Springer, 1999. doi:10.1007/978-1-4612-0515-9.

14 Leah Epstein, Asaf Levin, and Gerhard J. Woeginger. The (Weighted) Metric Dimension of Graphs: Hard and Easy Cases. Algorithmica, 72(4):1130-1171, 2015. doi:10.1007/ s00453-014-9896-2.

15 Melodie Fehr, Shonda Gosselin, and Ortrud R Oellermann. The metric dimension of Cayley digraphs. Discrete Mathematics, 306(1):31-41, 2006.

16 Florent Foucaud, George B. Mertzios, Reza Naserasr, Aline Parreau, and Petru Valicov. Identification, Location-Domination and Metric Dimension on Interval and Permutation Graphs. II. Algorithms and Complexity. Algorithmica, 78(3):914-944, 2017. doi:10.1007/ s00453-016-0184-1.

17 M. R. Garey and David S. Johnson. Computers and Intractability: A Guide to the Theory of NP-Completeness. W. H. Freeman, 1979.

18 Frank Harary and RA Melter. On the metric dimension of a graph. Ars Combin, 2(191195):1, 1976. 
19 Sepp Hartung. Exploring parameter spaces in coping with computational intractability. PhD thesis, Fakultät Elektrotechnik und Informatik der Technischen Universität Berlin, December 2014.

20 Sepp Hartung and André Nichterlein. On the Parameterized and Approximation Hardness of Metric Dimension. In Proceedings of the 28th Conference on Computational Complexity, CCC 2013, K.lo Alto, California, USA, 5-7 June, 2013, pages 266-276, 2013. doi:10. 1109/CCC. 2013.36.

21 Stefan Hoffmann and Egon Wanke. Metric Dimension for Gabriel Unit Disk Graphs Is NPComplete. In Algorithms for Sensor Systems, 8th International Symposium on Algorithms for Sensor Systems, Wireless Ad Hoc Networks and Autonomous Mobile Entities, ALGOSENSORS 2012, Ljubljana, Slovenia, September 13-14, 2012. Revised Selected Papers, pages 90-92, 2012. doi:10.1007/978-3-642-36092-3_10.

22 Mark G. Karpovsky, Krishnendu Chakrabarty, and Lev B. Levitin. On a New Class of Codes for Identifying Vertices in Graphs. IEEE Trans. Information Theory, 44(2):599-611, 1998. doi:10.1109/18.661507.

23 Jeong Han Kim, Oleg Pikhurko, Joel H. Spencer, and Oleg Verbitsky. How complex are random graphs in first order logic? Random Struct. Algorithms, 26(1-2):119-145, 2005. doi: 10.1002/rsa. 20049.

24 Peter J Slater. Leaves of trees. Congr. Numer, 14(549-559):37, 1975.

25 Peter J. Slater. Domination and location in acyclic graphs. Networks, 17(1):55-64, 1987. doi:10.1002/net.3230170105.

26 Peter J Slater. Dominating and reference sets in a graph. J. Math. Phys. Sci, 22(4):445-455, 1988.

27 Simon Tippenhauer. On Planar 3-SAT and its Variants. PhD thesis, Freien Universität Berlin, 2016. URL: https://www.mi.fu-berlin.de/inf/groups/ag-ti/theses/download/ Tippenhauer16.pdf.

28 Rachanee Ungrangsi, Ari Trachtenberg, and David Starobinski. An Implementation of Indoor Location Detection Systems Based on Identifying Codes. In Intelligence in Communication Systems, IFIP International Conference, INTELLCOMM 2004, Bangkok, Thailand, November 23-26, 2004, Proceedings, pages 175-189, 2004. doi:10.1007/ 978-3-540-30179-0_16. 